Thélème. Revista Complutense de Estudios Franceses

ISSN-e: 1989-8193

http://dx.doi.org/10.5209/THEL.54864

\title{
Vie et littérature chez Abdelfattah Kilito
}

\author{
Anouar Ouyachchi* ${ }^{* 1}$
}

Recibido: 03/01/2017 / Aceptado: 27/02/17

Résumé. L'écrivain marocain Abdelfettah Kilito est sans aucun doute l'un des écrivains dont les récits illustrent le plus, au Maghreb, les rapports complexes et énigmatiques que la littérature entretient avec la vie. Mettant souvent en scène des personnages qui vivent dans la compagnie des livres, ses récits ne cessent de montrer de quelle manière la littérature agit sur les hommes et les transforme. Cependant, au-delà de cette puissance métamorphosante de la littérature qu'il cherche à thématiser, à travers des personnages fictifs, c'est la situation de l'écrivain lui-même, qui ne peut parler de la vie qu'en habitant les livres, que Kilito s'évertue à représenter dans ses récits.

Mots clés : Kilito, littérature, vie, savoir.

\section{Vida y literatura en los relatos de Abdelfattah Kilito}

Resumen. EL escritor marroquí Abdelfettah Kilito es sin duda uno de los escritores cuyos relatos ilustran más, en el Magreb, la relación compleja y enigmática que la literatura tiene con la vida. Poniendo, a menudo, en escena a personajes que viven en compañía de los libros, sus relatos no dejan de mostrar cómo la literatura afecta a los hombres y transfórmalos. Sin embargo, más allá de este poder metamorfosis de la literatura que trata de tematizar, a través de personajes de ficción, ésta es la situación del propio escritor, que no puede hablar de la vida sin estar viviendo dentro de los libros y reciclando sus conocimientos, que Kilito se esfuerza por representar en sus relatos.

Palabras clave : Kilito, literatura, vida, conocimiento.

\section{Life and literature in Abdekfattah Kilito's narratives}

\begin{abstract}
The Moroccan writer Abdelfettah Kilito is undoubtedly an author from the Maghreb whose narratives best illustrate the complex and enigmatic relationship between literature and life. Often portraying characters living in the company of books, his narratives constantly show how literature influences and transforms individuals. However, beyond this metamorphic power of literature which he seeks to thematize through fictional characters, the writer himself is immersed in this experience. He can speak of life only by inhabiting the books and recycling the knowledge that Kilito strives to represent in his narratives. Keywords : Kilito, literature, life, narrative, knowledge.
\end{abstract}

Sommaire : La problématique de la copie. Amour et littérature. Littérature et culpabilité.

Cómo citar: Ouyachchi, A., (2017). "Vie et littérature chez Abdelfattah Kilito ». Thélème. Revista Complutense de Estudios Franceses, 32(1), 85-94.

\footnotetext{
* Université Moulay Ismail (Meknès, Maroc). ouyachi70@yahoo.fr

1 Membre du Laboratoire de Recherche sur la Culture, le Genre et la Littérature (LaRCGL).
} 
Quand on évoque les rapports entre la vie et la littérature, on pense d'habitude à la manière dont des récits littéraires absorbent la réalité et la transforment en la soumettant à des exigences purement littéraires. Autrement dit, la modalité la plus simple du rapport entre vie et littérature serait celle d'une représentation littéraire du monde extérieur qui fonctionnerait à la manière d'un trompe-l'œil en créant un « effet de réel ». En reprenant les termes de Jean-Marie Schaeffer, on peut dire que la fiction littéraire cherche à créer un " modèle d'univers " susceptible d'apporter « profit » et « plaisir » au lecteur en recourant à l' « imitation » et à la « modélisation » (Schaeffer, 1999).

Cependant, le rapport entre vie et littérature peut se décliner d'une tout autre manière : façon d'être au monde et par rapport au monde, la littérature, si on veut bien sortir du cadre formaliste pour s'intéresser à des questions éthiques et sociales, s'avère être une activité qui tient un discours sur le monde et produit aussi de la connaissance à sa manière, c'est-à-dire selon des modalités qui n'ont rien à voir avec celles du discours épistémique.

D'ailleurs, cherchant à définir la nature des liens que la littérature entretiendrait avec la connaissance dans son livre au titre très révélateur, La connaissance de l'écrivain. Sur la littérature, la vérité et la vie, le philosophe Jacques Bouveresse cite ces propos de Robert Musil :

Dans la mesure où la création littéraire transmet une expérience vécue, elle transmet aussi une connaissance ; cette connaissance n'est certes pas du tout la connaissance rationnelle de la vérité (même si elle est mêlée avec elle), mais toutes les deux sont le résultat de processus orientés de la même façon, étant donné qu'il n'y a justement pas un monde rationnel et en dehors de lui un monde irrationnel, mais un seul et unique monde qui contient les deux choses (Bouveresse, $2008: 28)$.

Disons-le donc : nous ne sortons pas de la littérature de la même façon que nous y entrons. Au demeurant ceux qui y font leur entrée en sortent-ils un jour? Un personnage comme, David Copperfield de Charles Dickens, illustre parfaitement la façon dont la compagnie des livres contribue à faire de nous les individus que nous sommes : «nous lisons pour la vie $»^{2}$. Dans le même sens, Umberto Eco, s'interrogeant, dans un essai, sur les fonctions de la littérature, fait cette observation :

Les malheureux qui, unis en bandes errantes, tuent en jetant des pierres du haut d'une rocade ou en mettant du feu à une enfant, qui qu'ils soient, [...] en sont [...] arrivés là $[\ldots]$ parce qu'ils restent exclus de l'univers du livre et de ces lieux où, par l'éducation et la discussion, ils seraient touchés par les reflets d'un monde de valeurs qui provient des et renvoie aux livres (Eco, $2003: 13)$.

Contre les pulsions meurtrières et incontrôlables de l'homme, le livre aurait donc un pouvoir thaumaturge. Mais n'avait-on pas reproché à l'auteur des Souffrances $d u$ jeune Werther d'avoir mené plusieurs amoureux éconduits au suicide? Tant en bien qu'en mal, la littérature agit donc sur nous et nous transforme si bien qu'au sortir

2 C'est le titre d'un chapitre du livre de Martha C. Nussbaum (Love's Knowledge. Essay on philosophy and literature, 1990), « Reading for life », qui nous a inspiré cette formule. 
d'un livre nous ne sommes plus pareils à nous-mêmes. La littérature est ce par quoi se réalise l'avènement de l'Autre en Soi, le devenir Autre de l'homme.

Cela dit, quel est le rapport des questionnements que soulève cette problématique des liens entre la vie et la littérature avec l'écrivain marocain Abdelfattah Kilito ${ }^{3}$ ?

Il est indéniable que les récits de Kilito offrent une bonne illustration de l'agir de la littérature sur les esprits. Comme l'explique Foucault, à propos de La Tentation de Flaubert, on pourrait dire que l'écriture de Kilito « se constitue d'entrée de jeu dans l'espace du savoir : elle existe dans un certain rapport fondamental aux livres » (Foucault, 1983 : 106). Que ce soit au niveau de l'essai ou de la fiction, l'écriture de Kilito jaillit du dialogue avec des textes appartenant à des cultures différentes. C'est un travail d'archive. D'ailleurs, en lisant les récits de Kilito, nous avons l'impression d'être, à la fois ici et ailleurs, maintenant et autrefois, tant l'énonciation littéraire, dans son errance entre les livres, trace incessamment une ligne de fuite qui nous fait voyager dans le temps et l'espace. Chez Kilito, on voit bien que le monde est une sorte de bibliothèque borgésienne que les personnages ne cherchent à aucun moment à quitter.

La puissance métamorphosante de la littérature se décline, chez Kilito, sous plusieurs formes. Nous en aborderons essentiellement trois aspects : le premier est en rapport avec la problématique de la copie qu'on rencontre dans Le cheval de Nietzs$c h e^{4}$. Dans ce récit initiatique, nous insisterons particulièrement sur la façon dont un exercice scolaire, somme toute banal, comme celui de la copie, est récupéré par l'auteur pour montrer combien le contact avec les livres peut nous transformer. Quant au deuxième aspect, il nous permettra d'examiner la relation qu'établit souvent Kilito, dans ses récits, entre amour et littérature avant de nous arrêter, pou finir, sur le malaise et le sentiment de culpabilité que produit chez les personnages de Kilito l'exercice de la littérature.

\section{La problématique de la copie}

Dans Le cheval de Nietzsche, c'est à travers l'exercice scolaire de la copie que le narrateur entre en contact avec la littérature. Surnommé «le singe calligraphe » par l'un de ses professeurs, en raison de sa belle écriture, le personnage prend petit à petit plaisir à faire d'abord les punitions pour toute la classe, puis à transcrire les livres scolaires avant de passer à la copie de chefs-d'œuvre littéraires entiers.

Quelque bizarre que soit cette situation, on aurait pu la comprendre. Mais deux détails nous laissent perplexes : premièrement le narrateur est incapable de lire sans passer par la copie ; la seule façon pour lui de lire consiste à recopier. Deuxièmement, la copie, dans le récit, est une activité vitale si bien que le jour où le personnage, ayant perdu foi dans l'exercice et décide de l'abandonner, il tombe malade. Et ce n'est que lorsqu'il se remet à copier que ses " maux de tête " disparaissent « comme par enchantement».

3 Écrivain et universitaire marocain, Abdelfattah Kilito a publié une dizaine d'essais dont Les Séances (Sindbad, 1983), L'Auteur et ses doubles (Seuil, 1985), L'Eil et l'Aiguille (La Découverte, 1992). Il est aussi l'auteur d'un roman, La Querelle des images (Eddif, Casablanca, 1985), et de quelques recueils de récits.

4 Paru chez Le Fennec en 2007, le livre regroupe quatre récits : 1. Le cheval de Nietzsche, subdivisé en six récits (désormais C.N pour les citations) ; 2. En quête, subdivisé en quatre récits; 3. Mö̈ra; 4. Du balcon d'Averroès. 
Qu'est-ce que donc copier dans ce cas?

Un acte de lecture qui affecte le corps et à travers lequel « la chair du lecteur matérialise les manières dont ce dernier perçoit le monde du livre » (Waty, 2012). A force de soumettre son corps, de plier celui-ci et d'en faire un instrument au service de la copie, le narrateur se trouve mystérieusement frappé jusque dans sa chair par les textes qu'il transcrit. Cet engagement physique, que suppose et implique l'exercice de la copie, révèle la part du corporel qui se joue dans tout acte de lecture. Il y aurait même quelque chose de l'ordre du vampirisme dans la façon dont le narrateur éprouve dans son corps le besoin et l'urgence de copier des textes. Cependant, il s'agirait là d'une sorte de vampirisme inversé. En effet, tandis que Dracula métamorphose ses victimes en les mordant, le narrateur de Kilito ne mord à la littérature que pour en être transformé et se constituer comme un éternel captif amoureux qui ne peut résister à l'appel des livres. D'ailleurs, dans Le Cheval de Nietzsche on peut lire : «Exclu des livres, je ne savais plus que faire de moi-même, et je ne pouvais espérer d'aide de personne » (C.N : 30-31).

Cette nécessité de la littérature pour le narrateur et la façon dont le manque se répercute, physiquement, sur lui sont une nouvelle fois soulignées quand celui-ci ne parvient plus, à un moment donné, à lire. La découverte de l'écrivain égyptien Akkad en classe ayant bouleversé ses habitudes de lecture, il se contente d'emprunter des livres à la bibliothèque et de les rendre sans les avoir lus. Et fait assez étrange, à cette période, comme par hasard, le narrateur souffre d'un début de surdité. Il n'y a nul doute que Kilito nous met ici en présence d'une érotique où la lecture contribuerait non seulement à produire un « corps bouleversé » (Barthes, 1984 : 44), mais à provoquer aussi, sur le mode de la jouissance, une rupture dans l'univers du lecteur. Afin de mieux comprendre cette érotique de la lecture et la façon dont celleci se déroule dans Le cheval de Nietzsche, il est peut-être nécessaire de revenir à la distinction que fait Roland Barthes entre « jouissance » et « plaisir » :

Texte de plaisir : celui qui contente, emplit donne de l'euphorie ; celui qui vient de la culture, ne rompt pas avec elle, est lié à une pratique confortable de la lecture. Texte de jouissance : celui qui met en état de perte, celui qui déconforte (peut-être jusqu'à un certain ennui), fait vaciller les assises historiques, culturelles, psychologiques, du lecteur, la consistance de ses goûts, de ses valeurs et de ses souvenirs, met en crises son rapport au langage (Barthes, 1973 : 25-26).

Pour revenir à la copie, nous pensons que celle-ci a quelque chose à voir, dans Le Cheval de Nietzsche, avec cette « lecture désirante [...] conductrice du Désir d'écrire » dont parle encore une fois Barthes (Barthes, 1984 : 44). Sauf que ce désir d'écrire fait prendre conscience ici au narrateur de son aliénation. En effet, le jour où celui-ci songe à écrire ses propres livres, il sent qu'il n'est plus lui-même, qu'il est habité par une autre parole. Dès lors, écrire devient pour lui une sorte d'aventure dans un espace occupé jusqu'à la suffocation par le langage d'autrui. Pris dans l'univers des textes qu'il transcrit, le jeune copiste se trouve frappé du sceau d'une altérité constitutive au point qu'il devient étranger à lui-même et que son propre livre, à venir, se donne déjà à lire comme la promesse d'une parole qui ne peut fatalement que résonner comme un écho à d'autres textes antérieurs. Ce qui est ainsi remis en question, à travers l'acte de la copie, c'est la primauté même de l'idée d'une origine sur un plan ontologique (Kintzler, 2003). La place que le narrateur cherche à 
occuper, en tant que sujet écrivant, implique une nécessité de «s'absenter comme sujet parlant pour advenir comme scribe d'une parole qui est dès lors parole des autres » (Adert, $1996: 168)$ :

Ce fut en vain que j'essayai de relever le défi. Chaque jour, à mon réveil, j'ouvrais un cahier vierge et attendais que le miracle se produisît. Tout ce qui se présentait à moi, c'était des phrases de livres que j'avais recopiés. J'étais habité par les paroles d'autrui. Incrustées dans ma mémoire, elles constituaient une richesse encombrante dont je n'arrivais à me débarrasser (C.N : 30).

Par ailleurs, il faut dire que le plaisir que prend le narrateur à se livrer à l'exercice de la copie est entaché d'un malaise, en rapport avec la position des parents qui ne voient pas en quoi un exercice pareil peut être utile, si ce n'est à déformer le dos, à crever les yeux et à dévier du bon chemin, c'est-à-dire de l'essentiel et des vraies études qui devraient mener à la médecine. Mais ce malaise est aussi provoqué par la mauvaise réputation de la copie, généralement associée à la punition et à la paresse.

Quel sens donc le narrateur devra-t-il attribuer à l'exercice devenu, pour lui, une sorte de mal nécessaire?

C'est à ce niveau qu'intervient la question des savoirs dans le récit. Par ses doutes et ses interrogations, le personnage provoque incessamment un embrayage de la fiction sur le discours didactique. En effet, à partir de la façon dont il éprouve dans son existence, de manière confuse et pré-critique des questions liées à la copie, le narrateur, en quête de modèle, est renvoyé à tout un savoir littéraire qui vient se fondre dans le récit. Les livres affleurent par ci et là, mais leur apparition n'en est pas moins motivée grâce à des personnages qui vont jouer un rôle important dans l'initiation du « jeune copiste » : ses professeurs qui tiennent lieu de « transmetteurs de savoir ». C'est ainsi que nous aurons dans le récit des fragments d'histoire littéraire arabe aussi bien qu'occidentale : Montaigne, le philosophe Alain et André Maurois sont alors cités, mais aussi ces anciens lecteurs/copistes de la culture arabe, l'Andalou Ibn Abd Rabbih, Jahîz, Abou Nouwas et d'autres.

C'est donc à partir de la question de la copie, dans sa relation avec la lecture et l'écriture, que le savoir investit Le Cheval de Nietzsche et transforme le récit en un intertexte où Kilito, outre sa passion narrative de conteur, satisfait aussi son activité de chercheur et d'essayiste. L'auteur emprunte tout un savoir à l'histoire littéraire qu'il redistribue de façon dramatique, dans son récit, pour nous montrer comment un individu particulier peut éprouver directement, dans son vécu, une question qui semble à priori être d'ordre philosophique. Autrement dit, Le Cheval de Nietzsche nous révèle comment des modèles littéraires sont mis à l'épreuve, à travers leur irruption dans la vie d'un personnage pour qui la vie et la littérature sont intimement liées.

\section{Amour et littérature}

Le deuxième thème autour duquel vient s'articuler la capacité de la littérature à nous transformer est celui de l'amour. En effet, dans les récits de Kilito, le rapport amoureux à la femme est souvent pensé à travers la mise en scène de personnages qui ne peuvent déchiffrer le monde et accéder à son sens que par le biais de la littérature. 
Dans Le Cheval de Nietzsche, il est aisé de remarquer la façon dont le thème de l'amour devient vite indissociable d'une réflexion sur la poésie arabe ancienne qui engendre une irruption des savoirs dans l'univers du récit et un embrayage de la fiction sur l'essai. Jouant sur le paradigme langue/femme/poésie, Kilito montre comment son narrateur, amoureux de Mlle Laurencin, ne peut s'empêcher d'associer l'amour à l'exercice de la poésie dans la langue de celle qu'il aime, c'est-àdire le français. Inversant le schéma qu'on rencontre, par exemple, dans un roman comme La Modification de Michel Butor qui veut que l'on pénètre dans une culture étrangère par le biais de la femme qui tient lieu de fil d'Ariane, le narrateur de Kilito cherche plutôt à conquérir la femme étrangère à partir de la langue de celle-ci et de sa littérature. Et c'est la raison pour laquelle il y a échec dans le récit, à notre sens. Non pas de l'amour qui n'a, de toute façon, qu'une valeur anecdotique ici puisque Mlle Laurencin, professeur de français, est beaucoup plus âgée que le narrateur, mais plutôt de la littérature. En effet, asservie à l'expression de la réalité dans ce qu'elle a de circonstanciel et de contingent, celle-ci devient provisoire et perd sa nécessité. D'ailleurs, quand Mlle Laurencin rentre en France, le narrateur, sans pour autant perdre définitivement l'envie de composer des poèmes, cesse d'en écrire. À travers ce rapport problématique que le personnage entretient avec la poésie, Kilito vise certainement à mettre en fiction, dans sont récit, deux positions théoriques concernant les rapports entre vie et littérature. Ainsi, si la tentative du personnage d'arrimer la poésie à la vie ordinaire et prosaïque s'inscrit dans une conception hétérotélique de la littérature, l'échec de son entreprise trouve son fondement dans une tradition littéraire idéaliste où la relation entretenue avec l'œuvre littéraire demeure plutôt « religieuse » et non « sécularisée » (Bouveresse, 2008). Il y aurait une sorte de tension entre la volonté d'exploiter littérairement les expériences les plus ordinaires et le refus d'asservir la littérature à l'expression du quotidien.

Cette association de la femme à la littérature se retrouve également dans le récit

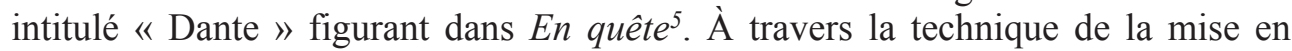
abyme, Kilito montre dans ce récit, comme dans « Duo », la façon dont les personnages cherchent à reproduire des modèles littéraires. Le passage décrivant la mâ̂tresse de maison se penchant pour regarder une page de la version italienne de $L a$ Divine Comédie, que le narrateur ne parvient pas à déchiffrer, constitue la reproduction d'une scène du cinquième Chant de L'Enfer, telle que le narrateur se la représente : la scène où Francesca et Paolo tombent amoureux l'un de l'autre en lisant l'histoire de Lancelot et la reine Guenièvre. D'ailleurs, ce n'est pas un hasard si le nom de la maîtresse de maison, dans le récit de Kilito, se réduit à l'initiale $\mathrm{F}$. qui évoque le nom de Francesca, et si le maître de maison «boite de la jambe droite » comme boite le mari de Francesca, Gianciotto de Rimini, prince laid et difforme. Ce sont là autant d'indices qui permettent à la réalité de se refléter dans le miroir de la littérature. Quelque paradoxal que cela puisse paraître, le cinquième Chant de $L ' E n f e r$, qui vient nourrir ici l'imagination, les fantasmes et le désir du narrateur, est une illustration des pouvoirs maléfiques de la littérature : si Francesca et Paolo se sont retrouvés condamnés à séjourner dans le deuxième cercle de l'Enfer, réservés

Paru initialement chez Fata Morgana en 1999, avant d'être réédité au Maroc par Le Fennec, En quête se compose de quatre récits : « Duo », « Dante », « Clefs » et « La bibliothèque ». 
à ceux qui ont commis le péché de la chair, c'est parce qu'ils ont été influencés par la lecture de l'histoire de Lancelot et la reine Guenièvre.

La littérature constitue donc ce par quoi le mal peut survenir ; elle fait perdre l'innocence et conduit en Enfer. Faisant fi de cette constatation, le narrateur de Kilito cherche, lui aussi, à séduire la maîtresse de maison par la littérature, à se plier à un modèle littéraire si maléfique soit-il.

Pourquoi cet attachement des personnages à la littérature quand bien même elle semble leur dire qu'elle peut les ruiner et causer leur perte?

Nous serions portés à dire que derrière cet acharnement à vivre dans et par la littérature, c'est une menace lourde d'implications métaphysiques qui se profile : l'angoisse du vide ou du néant. Le monde étant souvent évacué des préoccupations des personnages de Kilito, c'est la littérature qui viendrait leur servir de monde et de terre d'asile. La lecture constituerait avant tout, pour eux, une occasion de lutter contre la déliaison et la solitude qui les menacent; ils se mesurent à des êtres de papier qui, par un effet de miroir, leur rendent une certaine image d'eux-mêmes et leur permettent d'acquérir de la consistance. Aussi émergent-ils des ténèbres d'un monde qui, de toute façon, n'existe que pour être occulté et englouti par les livres. Si l'on peut formuler un cogito qui résumerait bien la situation des personnages de Kilito, ce serait bien celui-ci : « je lis, donc j'existe ».

Mais n'es-ce pas là une façon de sceller le clivage entre la vie et la littérature alors que toute l'entreprise littéraire de Kilito vise plutôt à fondre les deux et à brouiller les frontières entre elles ? L'intérêt que portent les personnages, dans les récits, à la littérature est révélateur de la position de l'écrivain quant à ce que peut apporter la littérature à l'expérience humaine. Nous pensons que Kilito serait tout à fait d'accord avec cette façon dont Martha Nussbaum, ayant toujours plaidé pour un usage pratique et moral de la littérature, cherche à redéfinir les rapports entre vie et littérature :

La littérature, dit Martha Nussbaum, est une extension de la vie non seulement horizontalement, mettant le lecteur en contact avec des événements ou des lieux ou des personnes ou des problèmes qu'il n'a pas rencontrés en dehors de cela, mais également, pour ainsi dire, verticalement, donnant au lecteur une expérience qui est plus profonde, plus aiguë et plus précise qu'une bonne partie des choses qui se passent dans la vie (Bouveresse, $2008: 31$ ).

En un certain sens, la littérature, dans plusieurs récits, est considérée comme une source de vérité. Dans « Dante » aussi bien que dans Moïra, un autre récit de Kilito, les personnages ont l'intime conviction qu'ils pourraient mieux connaître la femme et se rapprocher d'elle en accédant à sa bibliothèque et à ses lectures. Dans le chapitre intitulé « Pléiade » de La Querelle des images ${ }^{6}$, un texte de Kilito aux accents autobiographiques, on voit bien comment la littérature devient, pour le jeune Abdallah, un lieu où l'on part essentiellement à la rencontre de modèles qui nous renvoient notre image en nous faisant savoir que notre cas n'est pas unique : « La littérature l'attendait au tournant, il s'y référait pour savoir ce qu'il devait sentir, penser et faire, il s'y regardait désormais comme dans un miroir » (Q.I : 94).

6 Désormais Q.I. 


\section{Littérature et culpabilité}

Dans Le Cheval de Nietzsche, on retrouve un thème qui a déjà été abordé dans $L a$ Langue d'Adam, un essai de Kilito. C'est celui de la position de l'Islam par rapport à la poésie. En effet, le sentiment de culpabilité qu'éprouve le narrateur, dans « Le divan », quand il tombe amoureux de Mlle Laurencin et se met à composer des poèmes, est davantage lié au discrédit que jette le Coran sur le poète qu'à cet amour luimême. D'ailleurs, la pratique de la littérature et son exercice, quels qu'ils soient, engendrent souvent chez les personnages de Kilito un sentiment de culpabilité si bien qu'on ne peut pas dire qu'il existe des personnages heureux dans et par la littérature.

Nous avons déjà parlé du malaise du narrateur dans " Le singe calligraphe ». Mais dans « L'athlète » aussi, le rapport aux livres se déroule sous le signe de la culpabilité :

Chaque fois que je me présentais à la Bibliothèque, j'avais l'impression d'y pénétrer par effraction, Ali Baba au milieu des trésors immérités. Je me disais que je n'avais nul droit d'être dans la caverne ; à n'importe quel moment, les quarante voleurs pouvaient surgir et me châtier (C.N : 46).

Dans le récit intitulé «La Bibliothèque », figurant dans En Quête, et qui n'est pas sans rappeler Le Nom de la Rose d'Umberto Eco, et au-delà un certain rapport prométhéen au savoir, c'est par effraction que le personnage pénètre dans la bibliothèque qui garde jalousement ses livres serrés, les uns contre les autres. Dans La Querelle des images, la lecture des bandes dessinées est assimilée à un délit qui se déroule en cachette. Même dans le troisième récit d'En Quête, intitulé « Clefs », on peut dire que si le narrateur se rend quelque part coupable, aux yeux du lecteur, ce n'est pas parce qu'il porte tout son intérêt sur un manteau alors que sa mère, qui vient de mourir, attend d'être enterrée, mais parce qu'il est pris dans une urgence, celle de faire de la littérature, qui lui en fait perdre de vue une autre, ou si l'on veut l'essentiel. Comme dans Portrait d'un inconnu ou dans Martereau de Nathalie Sarraute, les narrateurs de Kilito, dans "Clefs » aussi bien que dans un récit comme Moïra, sont des écrivains en herbe qui ne peuvent pas s'empêcher de s'abandonner à une passion narrative, devenue incontrôlable pour eux, qui s'exerce en s'accrochant aux objets et aux détails comme les métonymes d'un sens, d'un monde ou de récits à construire.

Le cas de « la femme de $\mathrm{R}$ » dans La Querelle des images est d'ailleurs très significatif à cet égard. Ce personnage est indéniablement coupable en raison de sa curiosité qui donne souvent aux autres femmes « la désagréable impression d'avoir été fouillées et impitoyablement vidées de leur substance » (Q.I : 14). Mais au-delà de cette curiosité, c'est la passion narrative qui rend, à notre sens, « la femme de R » coupable : le jour où son mari, à qui elle racontait le soir les histoires recueillies pendant la journée, meurt, le personnage renonce à guetter les passants derrière la porte.

Cependant, si plusieurs personnages de Kilito vivent dans une inquiétude permanente, c'est parce qu'ils sont conscients, qu'aux yeux de leur entourage, ils ont perdu de vue l'essentiel. Dans leurs amours, dans leurs rapports aux autres aussi bien qu'au monde, les livres s'imposent et s'interposent tour à tour comme modèle ou écran, mais rarement comme lieu de rencontre. D'ailleurs, on peut voir dans $L a$ 
Querelle des images la façon dont les livres coupent du monde. Abdallah, devenu adulte, se voit, lors d'une visite à sa mère, brusquement saisi par l'urgence de lire une bande dessinée abandonnée sur le divan ; ce qui lui fait oublier le motif même de sa visite, c'est-à-dire le monde, c'est-à-dire encore une fois l'essentiel : « Zhor se leva, sans doute pour préparer le thé, peut-être aussi parce que connaissant la passion exclusive de son fils pour les images, elle savait que sa présence à ses côtés était pour un bon moment superflue » (Q.I : 55).

Ce qui est caractéristique des personnages de Kilito, contrairement à Don Quichotte, c'est une certaine lucidité sur eux-mêmes qui leur fait croire que la littérature ne peut pas remplacer le monde. En s'abandonnant à la lecture de la B.D, Abdallah « savait qu'il négligeait sa mère, lui préférant la compagnie d'êtres de papier. Vieux sentiment de culpabilité : enfant, il passait le plus clair de son temps à regarder des images, au lieu de faire ses devoirs » (Q.I : 56).

Umberto Eco explique que « certains personnages sont devenus en quelque sorte collectivement vrais parce que la communauté a fait, sur eux, au cours des siècles ou des années des investissements passionnels » (Eco, 2003 : 20). Aussi faudra-t-il « trouver un espace de l'univers où les personnages vivent et déterminent nos comportements, au point que nous les prenons comme modèles de vie, la nôtre et celle des autres » $(E c o, 2003: 21)$. Si ces propos peuvent s'appliquer parfaitement à l'écriture de Kilito, il n'en demeure pas moins qu'une telle vision du monde, incessamment ramené à la littérature, est à l'origine d'un paradoxe. En effet, aussi conviviale que soit le récit de Kilito, devenu lieu de rencontre et musée imaginaire où la connaissance est aussi, pour le grand plaisir du lecteur, une reconnaissance, pour le personnage, l'exercice de la littérature est, au contraire, tout sauf ce qui permet de tisser des liens avec autrui. À la communauté imaginaire de l'auteur et de ses lecteurs, réunis autour de lieux communs et d'habitudes culturelles, que fonde le récit, en répond certainement une autre : celle de personnages qui, étant fascinés par la copie, la lecture, la poésie, la passion narrative ou la bande dessinée, ont choisi de vivre avec des êtres de papier. Néanmoins, en mordant à la littérature, les personnages de Kilito consacrent, de façon irréversible, leur rupture avec le monde. Et c'est à partir de là qu'on peut d'ailleurs comprendre toute l'importance qu'accorde l'auteur au thème de la folie dans ses textes. Ses personnages, souvent rêveurs, timides et repliés sur euxmêmes, sont soupçonnés par les autres d'être des aliénés, des anormaux.

Cependant, au-delà des personnages, n'est-ce pas la situation même de tout écrivain que Kilito cherche à résumer par un effet de mise en abyme dans ses récits ? À vrai dire, la façon qu'a l'auteur d'écrire ses textes, en faisant systématiquement appel à d'autres textes, atteste, d'une certaine manière, qu'il n'y a d'écriture que celle qui célèbre la mort de l'écrivain au moment même où celui-ci cherche à proclamer sa propre naissance. Chez Kilito, l'écriture étant forcément reprise, citation et réécriture, l'écrivain se dissout dans l'acte même de l'écriture si bien que le récit s'érige en tombeau et que la signature sur la couverture du livre nous interpelle comme une épitaphe qui transforme la lecture en deuil. Cette disparition de la figure de l'écrivain, qui se retire derrière les textes, pour que ne subsiste, au final, qu'un livre, tramé à partir d'autres livres, institue le récit comme un lieu où la littérature se trouve saisie par la littérature. Si les personnages, dans les récits que nous avons analysés, sont de grands lecteurs ou des candidats à l'écriture, c'est qu'il n'y a de récit littéraire, au regard de Kilito, que celui d'une réflexion de la littérature sur ses conditions mêmes et sa propre possibilité. Il ne s'agit pas de raconter une histoire 
mais de thématiser, à travers des personnages, des questions liées à l'exercice de la littérature en mettant à nu les dispositifs qui président à l'acte créateur. Mais ce n'est pas pour autant que les récits de Kilito évacuent le monde et la vie de leurs préoccupations. D'ailleurs, est-il possible de les évacuer ? L'intérêt que prend la littérature pour les personnages et l'usage pratique qu'ils en font illustrent parfaitement la façon dont la lecture des récits littéraires peut nourrir la réflexion sur la question de la vie bonne. Car c'est de la vie et de la façon dont il faudrait la vivre qu'il s'agit au final. À vrai dire, nous ne nous mettons provisoirement à l'écart du monde en lisant que pour mieux réfléchir sur le monde et y retourner avec de nouveaux outils qui déterminent et influencent certaines de nos actions. Telle semble être l'idée que défend Kilito dans ses récits.

\section{Références bibliographiques}

Adert, L., (1996) Les mots des autres. Flaubert, Sarraute, Pinget. Paris, Presses Universitaires du Septentrion.

Barthes, R., (1984) « Sur la lecture », in Le bruissement de la langue. Essais critiques IV. Paris, Seuil.

Barthes, R., (1973) Le plaisir du texte. Paris, Seuil.

Bouveresse, J., (2008) La connaissance de l'écrivain. Sur la littérature, la vérité et la vie. Paris, Agone.

Eco, U., (2003) De la littérature. Paris, Grasset, coll. biblio-essais.

Foucault, M., (1983) « La bibliothèque fantastique », in Genette, G. \& T. Todorov (dir), Travail de Flaubert. Paris, Seuil, coll. points.

Kintzler, C., (2003) « La copie et l'original », in Revue Déméter [en ligne]. Décembre 2003, Université de Lille-3, disponible sur : http://demeter.revue.univ-lille3.fr/copie/kintzler.pdf [Dernier accès le 29 décembre 2016].

Nussbaum, M. C., (1990) Love's of Knowledge. Essays on philosophy and literature. NewYork/ Oxford, Oxford University Press.

Schaeffer, J.-M., (1999) Pourquoi la fiction? Paris, Seuil, coll., poétique.

Waty, B., (2012) «L'incarnation de la lecture : sensualisation et corporéité de lecteurs », in A. Milon \& M. Perelman (dir), Le livre au corps [En ligne]. Nanterre, Presses universitaires de Paris Nanterre, disponible sur : http://books.openedition.org/pupo/4283 [Dernier accès le 27 janvier 2017].

\section{Textes de Kilito}

Kilito, A., (1999) La Langue d'Adam et autres essais. Casablanca, Toubkal.

Kilito, A., (2000) La Querelle des images. Casablanca, Eddif.

Kilito, A., (2007) Le Cheval de Nietzsche. Casablanca, Le Fennec. 